\title{
Research on the influence of workpiece orientation during processing in disc centrifugal machine on the geometrical structure of its surface and processing efficiency
}

Jarosław PLICHTA, Mateusz JUNIEWICZ

DOI: 10.30464/jmee.2020.4.3.221

Cite this article as:

Plichta J., Juniewicz M. Research on the influence of workpiece orientation during processing in disc centrifugal machine on the geometrical structure of its surface and processing efficiency. Journal of Mechanical and Energy Engineering, Vol. 4(44), No. 3, 2020, pp. 221-226.

\section{VOLUME 4(44) | No. 3 | SEPTEMBER 2020}

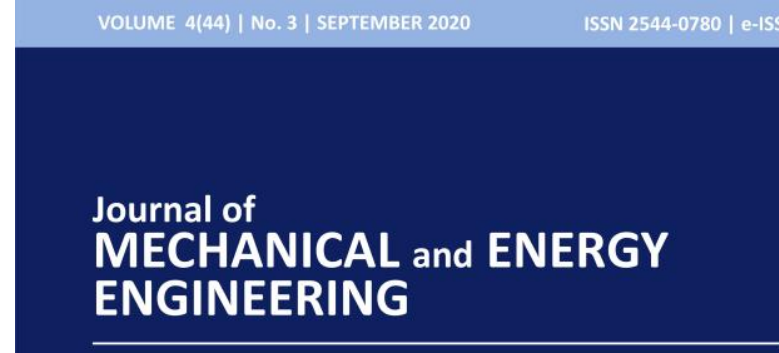

\section{Editor-in-Chief}

Waldemar Kuczyński

Editors

Wojciech Kapłonek | Krzysztof Nadolny
Journal of Mechanical and Energy

Engineering

Website: jmee.tu.koszalin.pl

ISSN (Print): 2544-0780

ISSN (Online): 2544-1671

Volume: $4(44)$

Number: 3

Year: 2020

Pages: 221-226

\section{Article Info:}

Received 10 September 2020

Accepted 21 September 2020

\section{Open Access}

This article is distributed under the terms of the Creative Commons Attribution 4.0 (CC BY 4.0) International License (http://creativecommons.org/licenses/by/4.0/), which permits unrestricted use, distribution, and reproduction in any medium, provided you give appropriate credit to the original author(s) and the source, provide a link to the Creative Commons license, and indicate if changes were made. 


\title{
RESEARCH ON THE INFLUENCE OF WORKPIECE ORIENTATION DURING PROCESSING IN DISC CENTRIFUGAL MACHINE ON THE GEOMETRICAL STRUCTURE OF ITS SURFACE AND PROCESSING EFFICIENCY
}

\author{
Jarosław PLICHTA ${ }^{1 *}$, Mateusz JUNIEWICZ ${ }^{1}$ \\ ${ }^{1 *}$ Faculty of Mechanical Engineering, Department of Production Engineering, Koszalin University of \\ Technology, Raclawicka 15-17, 75-620 Koszalin, Poland, e-mail: jaroslaw.plichta@tu.koszalin.pl
}

(Received 10 September 2020, Accepted 21 September 2020)

\begin{abstract}
The article describes the result of experiments which were intended to compare the results of different workpieces orientation in disc centrifugal machine. During experimental investigations, particular attention was paid to the optimal orientation the workpiece in working charge. The results of the research presented in this article confirm the benefit of horizontal orientation of the workpiece to the motion of the media in the working charge.
\end{abstract}

Keywords: disc centrifugal machine, geometrical structure

\section{INTRODUCTION}

During processing in disc centrifugal machine, the workpieces are placed loosely in rotating processing media. Their temporary position in relation to the processing media is random, which indeed enables surface treatment, but this process is not optimal. There is a relatively high percentage of the media sliding, rolling and scratching the surface with a relatively small percentage of micro-cutting. The energy of the processing charge is used to a limited extent to remove surface irregularities formed in the previous operations. This is due to the different speeds of the individual abrasive media in the rotating processing charge and their loose interaction with the surface of the workpieces. The structure of the rotating processing charge is characterized by toroidal movement, which is variable in terms of speed and energy, resulting from the fact that media are firstly lifted towards wall of the processing bowl and subsequently they fall down after speed loss due to frictional resistance.

Moreover, as the research carried out at the Department of Production Engineering has shown, there is a differentiation of the energy distribution of the working charge in its shaped spatial structure at a given rotational speed [1, 2] (Fig. 2). As a result the surface treatment is characterised by a large variety of media interaction modes and a large energy diversification

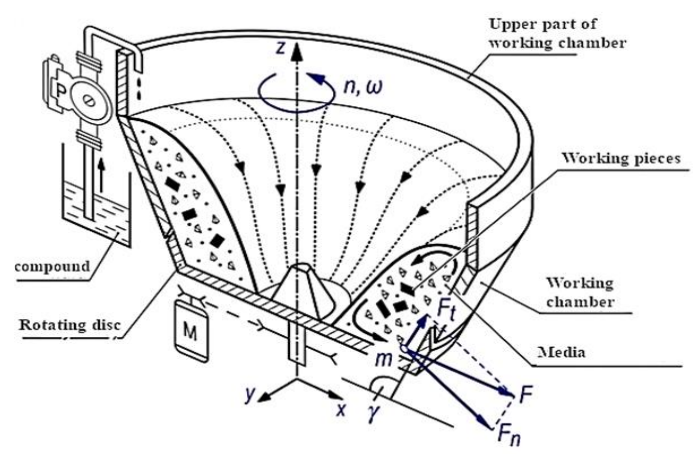

Fig. 1. The paths of movement of the abrasive media in the working container of the disc centrifugal machine $[1,2]$
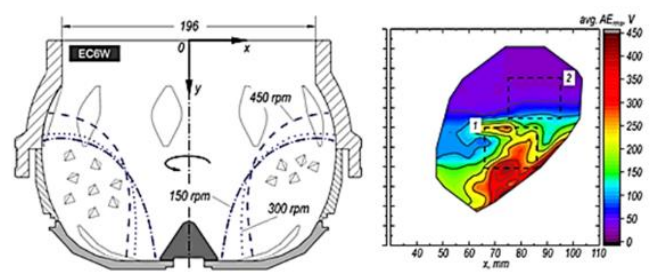

Fig. 2. The paths of movement of the abrasive media in the working container of the disc centrifugal machine $[1,2]$ 
Considering the above, the finishing process should be improved in order to use the energy of the processing charge in a more efficient way. One of such methods is specific orientation of the workpiece in a working holder, what allows to accumulate the abrasive media energy on a given surface of the workpiece and in result leads to increased process efficiency. It may be particularly advantageous if the workpiece is located in the area of the highest energy level $[3,4]$.

Works in this scope are carried out at the Department of Production Engineering of the Koszalin University of Technology, taking into account the workpiece holder with 4 degrees of freedom, ensuring a specific orientation of the workpiece in the working charge. Their essence is to determine the optimal processing position in relation to the finishing efficiency and the quality of the finished surfaces.

\section{METHODOLOGY OF EXPERIMENTAL RESEARCH}

The aim of the research was to determine how the orientation of the workpiece in the working charge influences the efficiency of surface finishing and the geometric structure quality of its surface.

The tests were carried out in disc centrifugal machine EC6 manufactured by Zakład Mechaniki Maszyn AVALON Polska (Fig. 3).

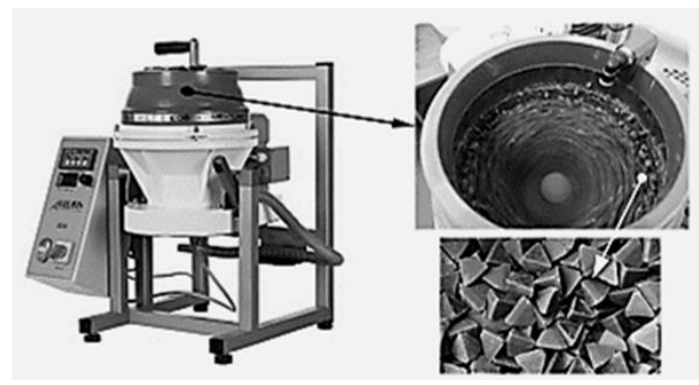

Fig. 3. EC6 rotary container smoothing machine

The workpiece orientation in the working charge was carried out by use of a holder with 4 degrees of freedom, in the area of the highest kinetic energy potential for a given rotational disc speed of the disc centrifugal machine. This holder allows the angular rotation of the workpiece in relation to the direction of the processing media rotary movement in the vertical orientation $(\alpha)$ and its angular inclination in the horizontal orientation $(\beta)$. It is also possible to combine these two orientations $(\alpha / \beta)-$ Fig. 4.

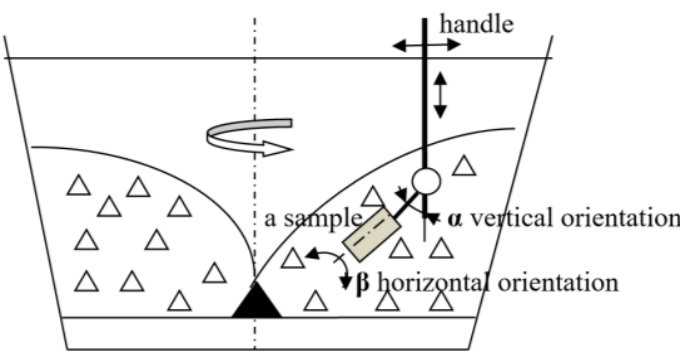

Fig. 4. Possibilities of workpiece orientation in the working zone of the disc centrifugal machine

Such design of the holder enables the workpiece to be moved in the axial section of the working charge, allowing it to be placed in the area of the highest working charge energy and providing 2-axis angular orientation (Fig. 5).

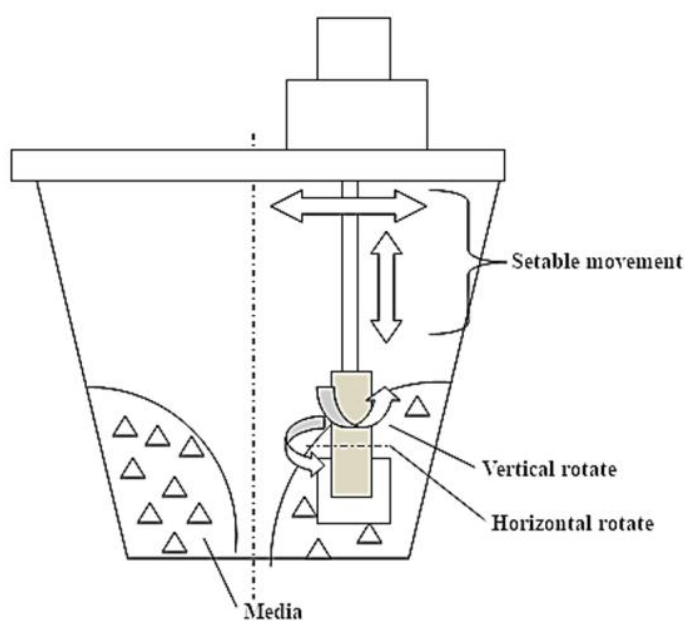

Fig. 5. Fig. 5. Orientation of samples in the working charge: a) vertical, b) horizontal

The obtained results were related to finishing of the workpiece without orientation, placed loosely in a rotating working charge.

The tested workpieces were flat-parallel shaped with dimensions of $20 \times 30 \times 2 \mathrm{~mm}$, made from PA11 aluminum alloy. The resin chips 02PP15 (nomenclature by AVALON) were used as the working charge. The treatment was carried out at the disc speed of $300 \mathrm{rpm}$. Duration of the finishing process was 15 minutes. The use of samples with relatively low hardness facilitated the mapping and traces observation of the impact of abrasive blocks on the treated surface. The research program is presented in Fig. 6. 


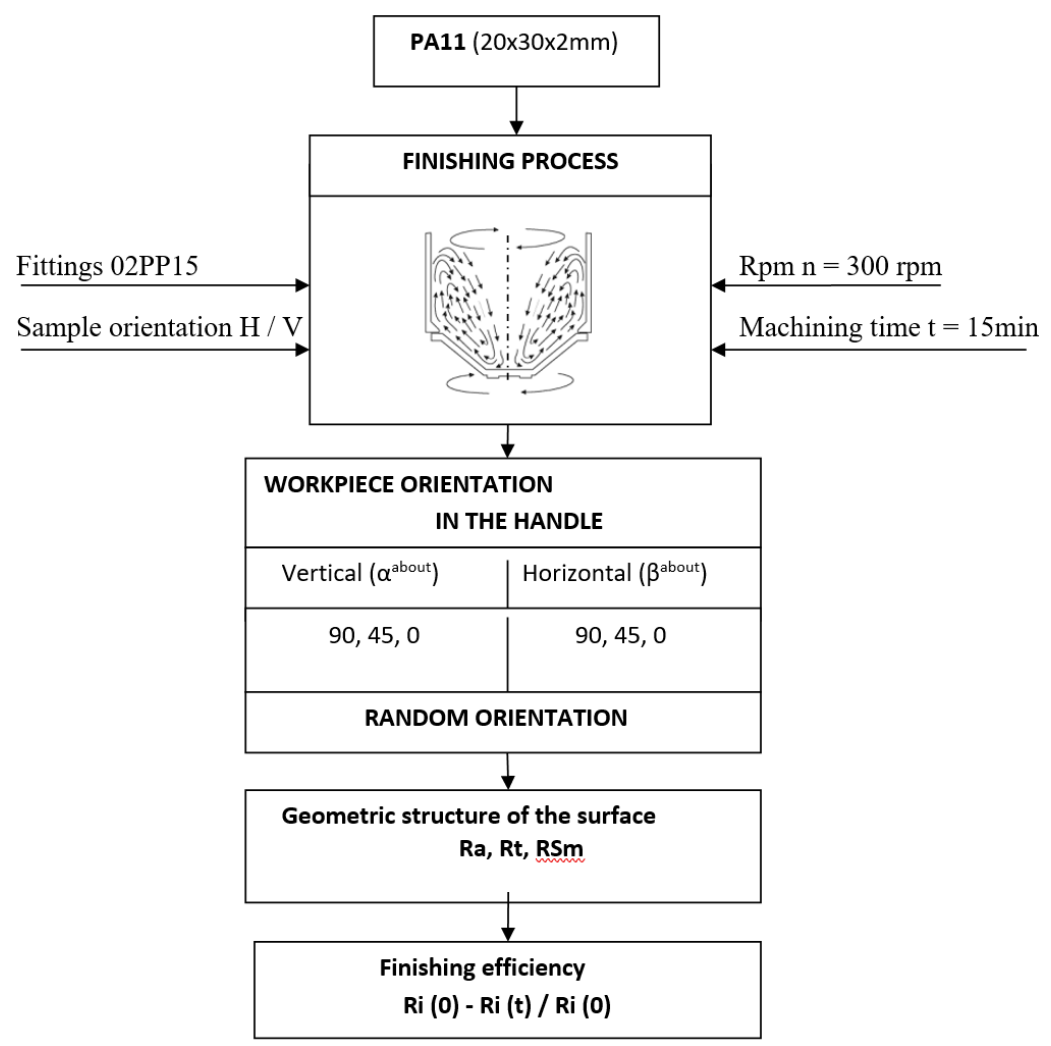

Fig. 6. Diagram of the research program

The results of the conducted tests confirm that in relation to the tested PA11 alloy workpieces, the intensity of the finishing and in result the geometric structure of the treated surface depend significantly on the vertical orientation of the object in relation to the direction of the work charge movement. This applies as well to the position of an object in a vertical, horizontal, angular and torsional position.

In case of perpendicular positioning of the workpiece in relation to the direction of the media movement, there is visible a relatively greater energy of their impact on the treated surface than in case of the horizontal positioning. This results in higher efficiency of finishing. The surface structure of the workpiece is also different, it is characterized by a greater degree of development, which is the result of increased number of finishing traces in form of craters and recessed areas. In case of angular orientation of the workpiece the processing intensity is lower, however higher than in case of horizontal orientation. In these two cases, the geometric structure of the treated surface is significantly smoothed (Fig. 7).

Whereas the angular orientation 450 in relation to its own axis, in each of these three cases, does not make significant difference both in terms of the finishing intensity and of the geometric structure shaping of the treated surface
In quantitative terms, the influence of all tested workpiece orientations on elementary mechanisms and the smoothing efficiency is presented in Table 1-3. It covers three basic cases (Fig. 8):

- perpendicular orientation of the tested surface in relation to the media movement direction a),

- parallel orientation of the tested surface in relation to the direction of media movement direction $b$ ),

- torsional orientation of the tested surface in relation to the direction of media movement direction c).

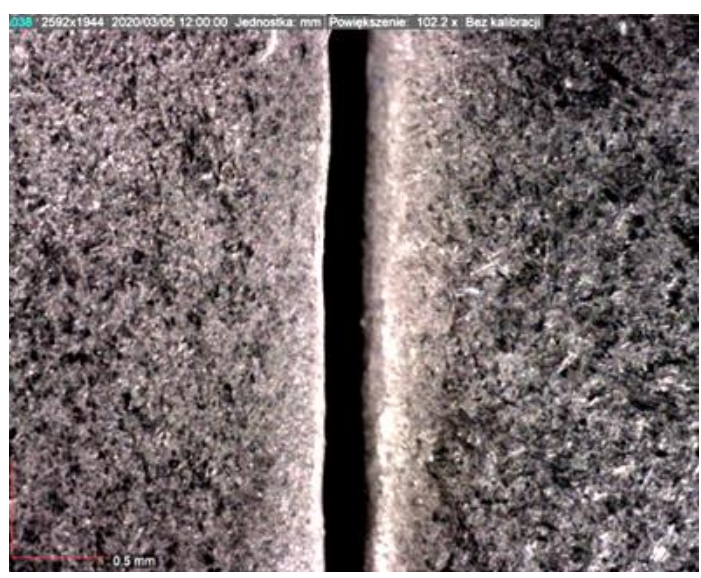

Fig. 7. Edge rounding of samples made from PA11 aluminium alloy after 15 min of smoothing: 
a) horizontal orientation, b) vertical orientation (magnification $\times 100)$
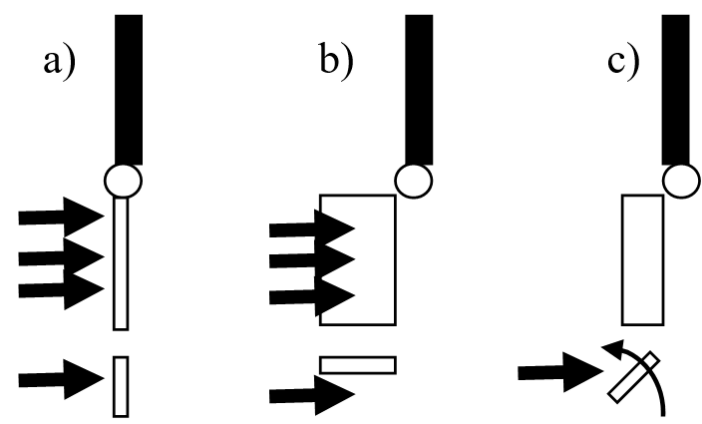

Fig. 8. Types of workpiece surface orientation in a vertical arrangement

Based on these analyzes, it can be concluded that the use of a different orientation of the workpiece during finishing the disc centrifugal machine has a significant impact on the mechanisms of shaping the geometric structure of the treated surface. This is due to the impact direction of abrasive media on it and the associated participation of individual elementary features occurring in this process.

And so, in the case of operating the abrasive media in the direction perpendicular to the surface, the main shaping mechanism are local craters, which result from media impact points. The structure of such surfaces is characterized by high isotropy, but also by relatively highest level of roughness. On the other hand, in case of media movement in the direction parallel to the finished surface, directed processing traces begin to dominate, resulting from micro-cutting and grooving, which ensures the lowest surface roughness for all tested angular orientations in the horizontal system. There is a visible orientation of the surface, it is not fully stochastic and is locally differentiated. On the other hand, the SGP shaping mechanism in a mixed system is more complex, in which the object takes the same positions as in the two previous systems and is additionally rotated by an angle of 450 . In this case the advantage is the favorable accumulation of the media's toroidal motion and the rotational motion. On the other hand, in case of an inclined workpiece orientation, along with the increase of the inclination angle, the surface roughness decreases with the lowest intensity.

These features are additionally overlapped by the toroidal movement of the fittings in the working charge (Fig. 1), which affects the directions of media movement in local areas. However, the primary influence on the SGP shaping is the rotational movement of the media, which is characterized by a much higher kinetic energy.

What is is even more interesting, for all tested horizontal and angle orientations in relation to its axis, the lowest values of roughness parameters occur for workpiece in the horizontal orientation (Fig. 9). This is probably the result of the beneficial effect, which base on the synergy of the rotary motion and the internal toroidal motion of the working charge.

Tab. 1. The dominant process features occurring when finishing the workpiece in the holder with an angular orientation perpendicular to the direction of the media movement (a)

\begin{tabular}{ll}
$R a=1.15 \mu \mathrm{m}$ & $\begin{array}{l}\text { A large number of craters (depressions) of significant } \\
\text { surface density and varied depth. Relatively few } \\
\text { scratches resulting from the micro-cutting process. } \\
R S m=0.159 \mathrm{~mm}\end{array}$ \\
$\begin{array}{l}\text { Surface structure with a high degree of isotropy. } \\
\text { Relatively high surface roughness. }\end{array}$ & $\begin{array}{l}\text { Decreasing number of craters, increasing number of } \\
\text { micro-nicks and scratches. Progressive smoothing of the } \\
R t=10.95 \mu \mathrm{m}\end{array}$ \\
$R S m=0.121 \mathrm{~mm}$ & $\begin{array}{l}\text { surface. The degree of surface anisotropy increases. } \\
\text { Lowering the surface roughness. }\end{array}$ \\
$R t=0.54 \mu \mathrm{m}$ & $\begin{array}{l}\text { A large number of scratches resulting from micro-cuts } \\
\text { and many marks resulting from grooving, rubbing and } \\
\text { rolling on fittings. Increase in the degree of anisotropy of } \\
\text { the surface. Significant smoothing and a decrease in } \\
R S m=0.097 \mathrm{~mm}\end{array}$ \\
\hline
\end{tabular}


Tab. 2. Dominant process features occurring when finishing the workpiece in the holder with an angular orientation parallel to the direction of the media movement (b)

$\begin{array}{ll}R a=1.12 \mu \mathrm{m} & \begin{array}{l}\text { A large number of scratches and traces of micro-cuts with } \\ \text { significant surface compaction and varied depth. Relatively } \\ R S m=0.145 \mathrm{~mm} \\ \text { few craters and depressions. Surface structure with a high } \\ \text { degree of anisotropy. Relatively high surface roughness. }\end{array} \\ R a=0.55 \mu \mathrm{m} & \begin{array}{l}\text { Decreasing number of craters, increasing number of micro- } \\ \text { nicks and scratches. Significant surface smoothing. } \\ \text { Increase in the degree of anisotropy of the surface. Light } \\ \text { structure orientation. }\end{array} \\ R a=0.12 \mu \mathrm{m}\end{array}$

Tab. 3. The dominant process features occurring during finishing of the workpiece in the holder with a torsional orientation in relation to the direction of media movement (c)

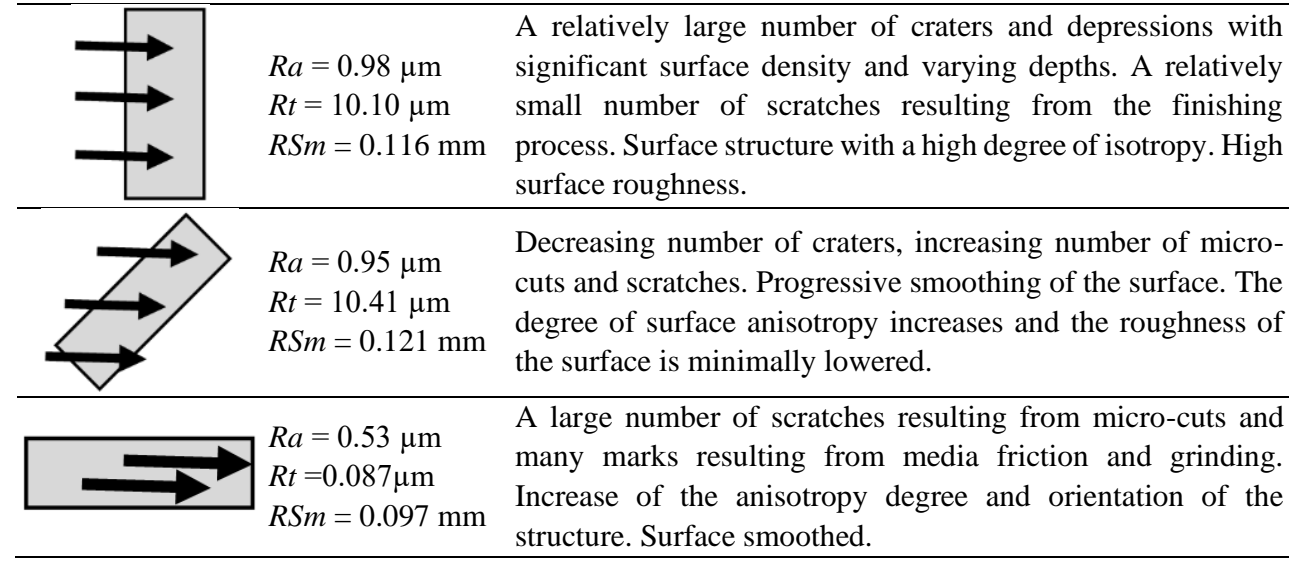

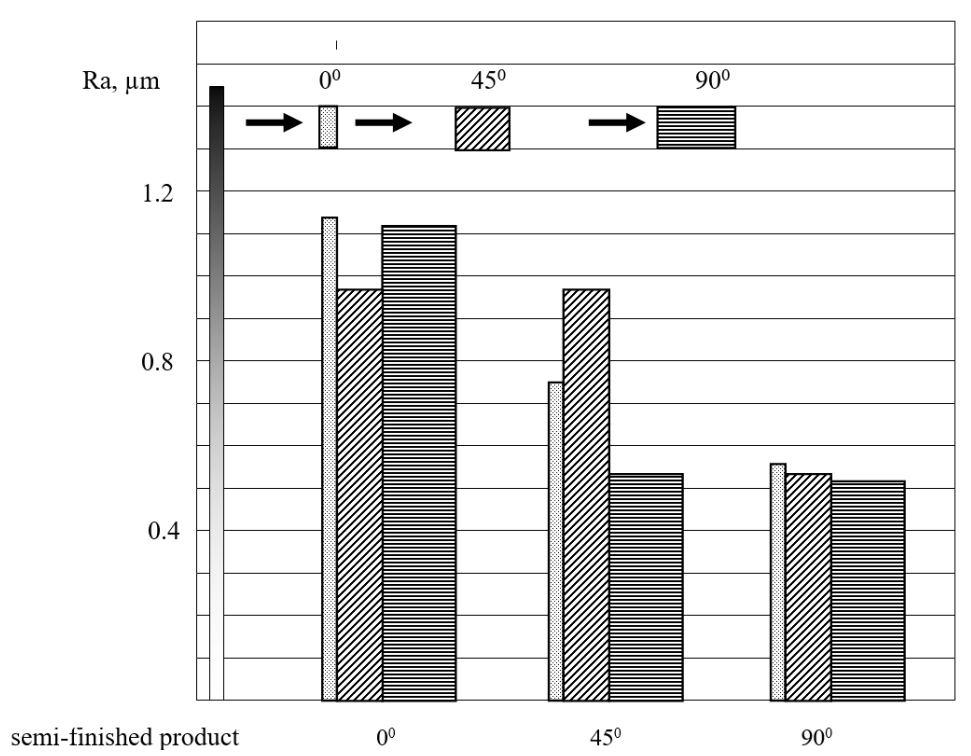

Fig. 9. The influence of the sample inclination angle in the horizontal system and the angle of its rotation in relation to the axis on the shaping of the $R a$ roughness parameter in the smoothing process 


\section{CONCLUSIONS}

By analysing all tested methods of workpiece orientation in the disc centrifugal machine, we can indicate for each of them the one, that ensures the lowest surface roughness of the tested samples

Despite the similar values of the $\mathrm{Ra}$ parameter, these profiles are characterized by a different degree of development and of smoothing, which results from different shares of individual process features. In this case, the main role is played by the elementary features related to micro-cutting and cratering, related to the orientation of the treated surface in relation to the direction of media movement.

The relative efficiency of smoothing surfaces shaped by these methods was determined on the basis of the following relationship:

$$
\Delta R a=\frac{R a(0)-R a(15)}{R a(0)},
$$

where: $R x(0)$ - the parameter of roughness of the output surface after printing, $R x$ (15) - surface roughness parameter after machining.

Tab. 4. Parameter values Ra for the relative cumulative angular set-ups of perpendicular machined surfaces and torsional settings

\begin{tabular}{cccc}
\hline $\begin{array}{c}\text { Torsion } \\
\text { settings } \\
\text { about the } \\
\text { axle }\end{array}$ & \multicolumn{4}{c}{ Angle settings in a horizontal position } \\
\cline { 2 - 4 } & $0^{\circ}$ & $45^{\circ}$ & $90^{\circ}$ \\
\hline $0^{\circ}$ & $\Delta R a=0.23$ & $\Delta R a=0.48$ & $\Delta R a=0.63$ \\
\hline $45^{\circ}$ & $\Delta R a=0.33$ & $\Delta R a=0.35$ & $\Delta R a=0.63$ \\
\hline $90^{\circ}$ & $\Delta R a=0.23$ & $\Delta R a=0.62$ & $\Delta R a=0.64$ \\
\hline
\end{tabular}

This shows quantity wise the intensity of lowering the roughness of the treated surfaces, depending on their orientation with respect to the direction of media movement. The reduction of the $R a$ parameter is particularly noticeable with the horizontal orientation of the machined surfaces (900), regardless of the angle of their rotation with respect to their axis. This is the result of media movement contiguous to this surface. A similar level of processing intensity also occurs when the surface is angled at 450 in respect to its axis. This fact can be explained by the synergy of rotational and toroidal motion of the media in the working charge.

This knowledge can form the basis for optimized design of workpiece holders for use in disc centrifugal machine in order to increase processing efficiency and reduce processing time. However, these tests should be treated only as exploratory and related to the processing of plane-parallel samples. In case of workpieces with complex shapes, it will be desirable not only to seek their favourable orientation, but also to implement additional rotational movement of the workpiece. It will also be necessary to determine the finishing times, depending on the type of material being processed and the expected final surface roughness, its orientation and level of isotropy.

\section{References}

1. Sutowski P., Plichta J., Kałduński P.: Determining kinetic energy distribution of the working medium in a centrifugal disc finishing process- part 1: theoretical analis. The International Journal of Advanced Manufacturing Technology 2019.

2. Sutowski P., Plichta J., Kałduński P.: Determining kinetic energy distribution of the working medium in a centrifugal disc finishing process- part 2: experimental analysis with the use of acoustic emission signal. The International Journal of Advanced Manufacturing Technology 2019.

3. Srivastav S. et al.: Effect of Workpiece Orientation, Lubrication and Media Geometry on the Effectiveness of Vibratory Finishing of Al6061 MATE Web of Conferences 30.04001 (2015).

4. Vijayaraghavan V. et al.: State-of-the-art in experimental and numerical modeling.

5. of surface characterization of components in mass finishing process. International Journal Advanced Manufacturing Technology.

\section{Biographical notes}

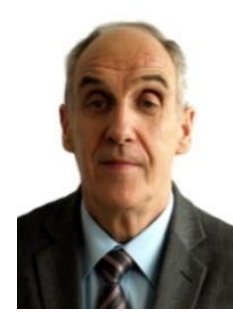

Jaroslaw Plichta received his M.Sc. degree in Mechanics and Machine Design and next Ph.D as well as D.Sc. degree and professor title in Machinery Construction and Operation, in 1976, 1981, 1997 and 2010, respectively. From 2006 to 2019 he was head of the Department of Production Engineering at the Koszalin University of Technology. His scientific interests focus on abrasive processes and tools, monitoring and diagnostics of machining processes as well as metrology. He has managing 4 national research projects, presenting results of his work at many international and national conferences, published more than 120 scientific papers in international and national journals, book chapters, as well as conference proceedings. He is also the author of 6 monographs, 4 academic textbooks and 11 national patents.

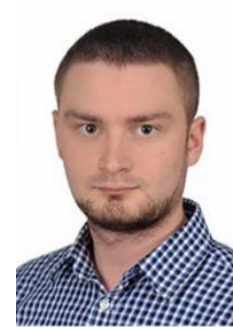

Mateusz Juniewicz received his M.Sc. degree in Mechanics and Machine design in 2017 at the Faculty of Mechanical Engineering of the Koszalin University of Technology. His diploma thesis entitled: "Modification of the rotary smoothing machine to vibration-stream treatment" was elaborated under the direction of Prof. Jarosław Plichta. 